\title{
To what extent have key recommendations from the Getting It Right First Time programme for urology in England been implemented?
}

\author{
William Gray ${ }^{1}$, Jamie Day ${ }^{1}$, Tim Briggs ${ }^{1}$, and Simon Harrison ${ }^{2}$ \\ ${ }^{1}$ NHS England and NHS Improvement London \\ ${ }^{2}$ Pinderfields Hospital
}

May 6, 2020

\begin{abstract}
Rationale, aims and objectives: The Getting It Right First Time (GIRFT) programme was set up to reduce unwarranted variation in healthcare practice and outcomes in England. The aim of this study was to investigate early changes in practice in urology based on the recommendations made. Key recommendations included: 1) to increase rates of day-case surgery for transurethral resection of bladder tumour (TURBT), 2) to reduce use of stenting as a primary procedure for emergency presentations with ureteric stones and 3) to reduce waiting times for male bladder outflow tract surgery following emergency presentation with urinary retention. Methods: Data on patient age, the treatment provider, dates of admission and discharge, diagnoses and procedures conducted were extracted from the Hospital Episodes Statistics database from January 2014 to December 2019. The dates of visits by members of the GIRFT team was taken as the intervention point. Interrupted time series analysis was used to identify trends pre- and post-intervention. Results: There was evidence of a significant increase in the proportion of patients seen as day-cases for TURBT and decreased use of stents and increased use of ureteroscopy or extracorporeal shock-wave lithotripsy on first presentation with ureteric stones following GIRFT visits. However, there was no significant change in waiting times for surgery to treat patients who had an emergency presentation with urinary retention. Conclusions: In the first 18 months following the last of the GIRFT visits, there is some evidence that the recommendations made are already having an impact on clinical practice. The reasons why some recommendation appear to be harder to implement requires further investigation.
\end{abstract}

\section{INTRODUCTION}

Health services must always look to work as efficiently as possible. Any suggested changes to clinical practice should aim to either improve outcomes for patients or to reduce financial and human resource use whilst maintaining outcomes for patients. ${ }^{1,2}$ The Getting It Right First Time (GIRFT) programme was set up in 2012 to minimise unwarranted variation in service provision and patient outcomes in the National Health Service (NHS) in England. The overall aim is to increase efficiency while maintaining good outcomes and experience for patients.

Unwarranted variation can be defined as any variation clinical practice which impacts on outcomes for patients which cannot be justified on the grounds of patient need, patient preference, equivocal evidence or intractable resource limitation. ${ }^{3}$ Examples of causes of unwarranted variation include clinician preference for a particular intervention, a lack of engagement with recent evidence, a lack of training in new techniques and poor resource allocation. ${ }^{3}$

The GIRFT methodology is based on engagement with clinicians by peers. During initial 'deep dive' visits to each health service provider (NHS Trust), practice and patient outcomes for the provider are reviewed relative 
to other providers and the data discussed in the context of local resource constraints, service organisation and the population served. Providers demonstrating good patient outcomes or efficient resource use are discussed as possible models for those with poorer outcomes or resource use. Key recommendations are fed back to providers with support for implementation of service improvement.

The GIRFT national report for urology was published in July 2018 and summarised the key findings for the programme. ${ }^{4}$ The report identified a number of areas where there appeared to be unwarranted variation in clinical practice across providers that impacted negatively on patient outcomes or cost-effectiveness and made a number of recommendations of ways to reduce such variation. These included: 1) increased use of day-case surgery for transurethral resection for bladder tumour (TURBT), 2) reduced use of ureteric stents as the primary intervention for emergency presentations with ureteric stones and 3) reduced waiting times to male bladder outflow obstruction surgery, for example transurethral resection of the prostate (TURP), from first emergency admission with urinary retention.

The aim of this study was to investigate the impact of the GIRFT programme on these three areas of practice. In the longer term it was hoped that this may lead to the identification of barriers and facilitators to implementation. We used interrupted time-series analysis (ITSA) to analysis data from the period before and after visits to each NHS trust by the GIRFT team with the recommendations.

\section{METHODS}

\section{Ethics}

This study used data from the Hospital Episode Statistics (HES) database for England. The HES database is collected by NHS Digital for all patients seen at hospitals in England. Permission to access the database was granted to the GIRFT programme working within the NHS Improvement information technology environment. Consent from individuals involved in this study was not required. The presentation of data follows current NHS Digital guidance for use of HES data for research purposes. ${ }^{5}$

\section{Data collection}

In England all NHS hospitals are organised into trusts. Each trust provides secondary and tertiary care for a geographically defined catchment population. Typically, each trust runs between one and four larger district hospitals depending on the size and spread of their catchment population and local service delivery models. HES data are collected from all NHS Trusts in England and related to episodes of hospital care provided by the NHS. The data are entered by trained data coders within each trust. We used data for the six-year period $1^{\text {st }}$ January 2014 to $31^{\text {st }}$ December 2019.

\section{Data extraction}

For all three procedures, data were extracted for patient age, the NHS trust providing the care, the method of admission (e.g. elective, day-case, emergency), the procedures conducted during the admission and any relevant diagnoses.

Day-case surgery for TURBT

Data were extracted from HES for all TURBT procedures conducted during the six year study period using the Office of Population Censuses and Surveys Classification of Interventions and Procedures version 4 (OPCS-4) code M421. Patients were then excluded if they were not an elective or day-case admission, TURBT was not the dominant procedure during the spell, their pre-operative stay was greater than one day, there was a subsequent TURBT for the same person within the study period or if the trust at which they were seen was not visited by the GIRFT team. Day-case admissions were identified where the patient was admitted and discharged on the same day.

Use of ureteric stents

Data were extracted for all patients that had been admitted to hospital as an emergency admission during the six year study period with a diagnosis of urinary tract stones using the International Statistical Classification 
of Diseases and Related Health Problems $-10^{\text {th }}$ revision (ICD-10) codes N201, N202, N209. Patients with kidney stones, bladder stones and stones in other areas of the lower urinary tract were not included in the dataset. Key medical procedures of interest during the admission were use of a ureteric stent (OPCS codes M274, M292), use of ureteroscopy (OPCS codes M271, M272, M273, M281, M282, M283, M288, M289) and use of ESWL (OPCS codes M141, M311). These data were then linked to any subsequent elective ureteroscopy or ESWL procedure conducted in the 30 days following the emergency admission, where a stent had not been fitted during the initial emergency admission.

Waiting time for male bladder outflow tract surgery

Data were extracted for all patients that had been admitted to hospital as an emergency admission with a diagnosis of urinary retention (ICD-10 code R33) from $1^{\text {st }}$ January 2014 to $31^{\text {st }}$ December 2018. Only first emergency admissions were included; subsequent emergency admissions with urinary retention for the same patient were excluded. These data were then linked to any subsequent bladder outflow tract procedure (OPCS codes M651, M652, M653, M654, M655, M658, M659) in the 12 months following the emergency admission. Only first procedures were counted for those that had more than one bladder outflow tract procedure in the 12 month period.

\section{Intervention}

The date of the visit to the trust by the GIRFT team where key recommendations were presented was taken as the key intervention point. Visits were spread over a 20 month period; the first visit was on $10^{\text {th }}$ August 2016 and the final visit on $19^{\text {th }}$ April 2018.

The time from the GIRFT visit to the hospital admission date for each patient was calculated in days. Admissions during the baseline period (before the GIRFT visit) had time recorded as a negative number and on the day of the visit recorded as time zero. Time was then divided into periods of 30 days with the day of the visit and the next 29 days coded as period 0 and the next 30 days as period one etc. Similarly, the 30 days prior to the visit were coded as period minus one and the previous 30 days as period minus two etc.

\section{Outcomes}

Day surgery for TURBT: Percentage of patients receiving TURBT as day-case surgery during each time period.

Use of ureteric stents: Percentage of patients fitted with a stent as their primary intervention on first emergency admission. Percentage of patients receiving ureteroscopy or ESWL as their primary procedure on first emergency admission or receiving elective ureteroscopy or ESWL procedure in the 30 days following the emergency admission, where a stent had not been fitted during the initial emergency admission.

Waiting time for male bladder outflow tract surgery: Waiting times for male bladder outflow tract surgery from first emergency admission with urinary retention during each time period.

\section{Data management and statistical analyses}

Data were extracted from and onto a secure, encrypted SQL server controlled by NHS Improvement. Data were analysed within this secure environment using standard statistical software: Microsoft Excel (Microsoft Corp, Redmond, WA, USA), Stata (Version 13, StataCorp LLC, College Station, TX, USA) and Alteryx (Alteryx Inc, Irvine, CA, USA).

ITSA was used to investigate changes in practice in response to each intervention for each recommendation. ITSA allows temporal trends in data to be examined prior to an intervention, any immediate changes at the point of the intervention and longer term changes in trend post-intervention to be examined. ${ }^{6}$ The itsacommand in Stata was used to conduct the analysis. ${ }^{7}$ The procedure used ordinary least squares to estimate the model coefficients with Newey-West standard errors used to produce confidence intervals for the estimates. 
Data were categorised into the 30-day periods outlined above for the analysis. For all three datasets, periods at the start and end of the time series with fewer than 200 admitted patients per trust were excluded. Periods with smaller numbers of patients at the start and end of the time series arose since the trusts visited first had a very long post-intervention period and the trusts visited last had a very long pre-intervention period. For the day-case surgery for TURBT dataset this involved removing 6 (3 at start and end) of 94 time periods. For treatment for the use of ureteric stents dataset this involved removing 11 ( 7 at start and 4 at the end) of 94 time periods. For the waiting times for TURP dataset this involved removing 22 (12 at the start and 10 at the end) of 82 time periods.

Autocorrelation was investigated using the Cumby-Huizinga general test

for autocorrelation and the test implemented using the actestcommand in Stata. ${ }^{8}, 9$ Autocorrelation was only detected for the TURBT dataset and was corrected for by a lag of one. For all other datasets and outcomes studied the lag period was set at zero.

\section{RESULTS}

\section{Day-case surgery for TURBT}

The data extraction process is summarised in Supporting Information 1, Supplementary Figure 1 . Data were available for 99,790 patients, $15,149(15.2 \%)$ of whom had day-case surgery. The mean age of patients was 72.5 (SD 11.595) before GIRFT visits and 72.3 (SD 11.751) after. Procedures were conducted across 125 trusts during the baseline pre-visit period. In the pre-visit period, in the 123 trusts seeing 50 patients or more during the study period, the mean percentage of patients receiving a TURBT as a day-case varied between $1.5 \%$ and $68.4 \%$ across trusts.

The change in the use of day-case surgery across the study period is summarised in Figure $\mathbf{1}$ and the ITSA presented in Table 1 . At the start of the study period an average of $10.2 \%$ of patients received TURBT procedures as day-case surgery. During the pre-GIRFT visit period this proportion rose by an average of $0.105 \%$ per 30 day period ( $1.3 \%$ per annum), after the GIRFT visit the increase in use of day surgery was almost three times as rapid, at $0.281 \%$ per 30 day period $(3.4 \%$ per annum). Both these trends were significant as was the change in trend ( $0.176 \%$ per 30 day period, $2.1 \%$ per annum).

\section{Use of ureteric stents}

The data extraction process is summarised in Supporting Information 1, Supplementary Figure 2. Data were available for 54,124 patients. The mean age of patients was 47.9 years (SD 17.449) before GIRFT visits and 48.5 years (SD 15.896) after. Procedures were conducted across 123 trusts during the baseline previsit period. In the pre-visit period, in the 121 trusts seeing 50 patients or more during the study period, the mean percentage of patients receiving a stent varied between $0 \%$ and $46.0 \%$ across trusts and ureteroscopy or ESWL use varied from $0.7 \%$ to $38.7 \%$. During the pre-visit period $6.4 \%$ of patients received ureteroscopy or ESWL during the first emergency admission and $4.3 \%$ at a subsequent elective admission in the following 30 days.

The change in the use of ureteric stents and ureteroscopy or ESWL across the study period is summarised in Figures 2 and 3respectively. The ITSAs for both outcomes are presented in Table $\mathbf{1}$. At the start of the study period an average of $12.2 \%$ of patients received a stent during their first emergency admission. During the pre-GIRFT visit period this proportion rose significantly by an average of $0.105 \%$ per 30 day period (1.3\% per annum), after the GIRFT visit stent use had no significant trend with time, declining by $0.044 \%$ per 30 day period. However, the change in trend from pre- to post-visit was significant at $-0.148 \%$ per 30 day period, (1.8\% per annum).

For use of ureteroscopy or ESWL to treat ureteric stones, at the start of the study period an average of $10.8 \%$ of patients received ureteroscopy or ESWL during their first emergency admission or electively within 30 days of this admission without first receiving a stent. During the pre-GIRFT visit period this proportion did 
not change significantly with time. After the GIRFT visit ureteroscopy or ESWL use increased significantly at a rate of $0.073 \%$ per 30 day period ( $0.9 \%$ per annum).

\section{Waiting times for male bladder outflow tract surgery}

The data extraction process is summarised in Supporting Information 1, Supplementary Figure 3 . Data were available for 23,798 patients. The mean age of patients was 73.1 years (SD 8.538) before GIRFT visits and 72.9 years (SD 8.533) after. Procedures were conducted across 125 trusts during the baseline pre-visit period. In the pre-visit period, in the 115 trusts seeing 50 patients or more during the study period, the mean waiting time before the GIRFT visits varied from 72.2 days to 184.9 days across trusts.

The change in waiting times for TURP across the study period is summarised in Figure 4 . The ITSA is presented in Table 1. At the start of the study period the average waiting time was 126.3 days. During the pre-GIRFT visit period this proportion rose significantly by an average of 0.310 days per 30 day period (3.7 days per annum), After the GIRFT visit there was no significant trend to either increasing or decreasing waiting times, suggesting some levelling off in waiting times, although the change in trend was not significant (decrease in the upward trend of 0.050 days waiting time per 30 day period; 0.6 days per annum).

\section{DISCUSSION}

The GIRFT programme is funded by the United Kingdom Department of Health. It aims to improve patient care through highlighting unwarranted variations in clinical practice. Clinical outcome data are presented to clinicians highlighting the performance of their trust relative to all trusts in England. Where unwarranted variation in outcomes is identified, clinicians and managers are engaged to identify ways in which outcomes could be improved. Ultimately, the approach relies on decision-makers within individual trusts recognising that their outcomes may be sub-optimal and then working constructively to improve. The use of data to benchmark performance and to identify subsequent improvements is key to this approach. Our study investigates the impact of the GIRFT programme on three specific recommendations from the GIRFT National Report in urology.

Use of day-case surgery for TURBT was increasing steadily prior to GIRFT visits, but the rate of increase more than doubled after the GIRFT visits. Based on our analysis, the GIRFT programme has had a significant impact, reducing use of hospitals beds for overnight stay. Our team have previous reported outcomes for TURBT performed as day-case surgery to be at least as good as those for TURBT performed with an overnight stay. ${ }^{10}$ Whilst not all patients undergoing TURBT will be suitable for day-case surgery, with some requiring an extended period of monitoring due to co-morbidity, frailty or disease severity, the increased use of day-case surgery for TURBT is encouraging. Nevertheless, with a number of trusts performing over half of all TURBT procedures as day-case, there appears to be significant scope for greater use of day-case surgery for many trusts.

For stent use to manage ureteric stones, we identified a significant temporal trend towards greater ureteric stent use prior to GIRFT visits and a significant change in trend after visits. We recognise that use a ureteric stent will be the best treatment option for some patients presenting with urinary tract stones, notably those with associated sepsis, and in these cases its use is clearly appropriate. However, complications associated with stenting are relatively common. ${ }^{11}$ Consequently, its use purely as a short-term measure, where other evidence-based, definitive treatment options are indicated and available, should be discouraged. ${ }^{12}$ During the same period, use of ureteroscopy or ESWL increased significantly after GIRFT visits. Such changes are likely to reduce the need for return admissions and so represent an efficient use of resources but are also likely to improve patient quality of life by offering definite treatment at an earlier time point.

Although there was a significant trend towards longer waiting times for male bladder outflow tract surgery after a presentation with urinary retention prior to GIRFT visits, which was not apparent afterwards, this change was not significant, suggesting any impact of GIRFT visits on waiting times was limited. The reasons why this recommendation was not implemented to the same extent as the other recommendations investigated are likely to be multifactorial, complex and vary from trust to trust. However, reducing surgery waiting times 
against a backdrop of increase pressure on services, due to factors such as demographic ageing and increasing morbidity of the background population, is likely to be challenging. ${ }^{13}$ Indeed, the steady and significant increase in outflow tract surgery waiting times during the baseline period provides evidence of this. Compared to the other recommendations investigated, reducing waiting times may be a more intractable problem that the changes to practice and service organisation required to move to a day-case model for TURBT or to reduce stent use for ureteric stones. Moreover, these other recommendations have the potential to either reduce costs, free-up beds or reduce human resource requirements. Reducing waiting times may only be achievable though increasing bed usage and staff numbers. Nevertheless, the GIRFT methodology is based on engagement with key stakeholders at a local level to help understand how recommendations can best be implemented within a given setting. ${ }^{14}$ Ongoing support is offered to trusts to help them implement recommendations. Further investigation of barriers and facilitators to implementation of GIRFT recommendations is needed. In other areas of healthcare, where barriers and facilitators to implementation of guidance has been investigated, resource constraints have been identified as a key barrier ${ }^{15,16}$ and engagement with clinicians locally, peer support and use of data to change perceptions were found to be facilitators. ${ }^{17}$

ITSA is a potentially powerful technique for the analysis of the impact of an intervention. ${ }^{18}$ It is particularly useful where a randomised controlled trial is not practical, and this is often the case when evaluating the impact of a new healthcare policy or quality improvement programme. ${ }^{19}$ However, one of the major threats to the validity of ITSA is that it is often not certain that it was the intervention of interest that caused any observed change. In our study this is less of an issue, since the visits to individual trusts were spread over 20 months, with each trust having its own intervention point. For the same reason the impact of seasonal factors is likely to be small, and the lack of any significant autocorrelation supports this view. A second threat to ITSA validity is changes in the way data are recorded over time. ${ }^{19}$ In our case, this is also unlikely to be a major problem since data are recorded by experienced data coders and there have been no major changes in the codes used over the time period covered. As such, our findings are likely to be relatively robust.

One further limitation of the ITSA approach we used is that there is no comparator group that received no intervention. We are unable to comment on how stent use would have changed without our interventions.

In summary, we present evidence that GIRFT programme recommendations around increasing rates of daycase surgery for TURBT and reducing stent use and increasing use of ureteroscopy or ESWL for ureteric stones have had an impact on clinical practice across England. The recommendation to reduce waiting times for male bladder outflow surgery have, as yet, had no significant impact on clinical practice. Further research should look to investigate reasons for good and poor adoption of recommendations and clinical guidelines within health services with a view to establishing the most effective methods of influencing practice. Although the GIRFT methodology may have helped to remove a number of barriers to effective implementation of changes to clinical practice, better understanding how the methodology can be adapted to address more intractable issues is needed.

\section{Conflicting interests}

The authors declare that there is no conflict of interest.Funding

This research received no specific grant from any funding agency in the public, commercial, or not-for-profit sectors.Informed consent

Informed consent was not sought for the present study because it was an analysis of routine clinical data.Ethical approval

Ethical approval was not sought for the present study because it did not directly involve human participants. This study was completed in accordance with the Helsinki Declaration as revised in 2013.

\section{Contributorship}


This study was designed and organised by SH, JD, TB and WKG. Data cleaning, analysis and writing of the first draft was by WKG, supported by JD and SH. All authors critically reviewed the manuscript and agreed to submission of the final draft.

\section{Information Governance}

This report does not contain patient identifiable data. The data in this report are anonymised. Request for any underlying data will not be granted as the data are calculated from data under licence/ data sharing agreement from NHS Digital and/ or other data provider where conditions of use (and further use) apply. Copyright (C) 2013, 2014, 2015, 2016, 2017 re-used with the permission of the Health \& Social Care Information Centre. All rights reserved.Acknowledgements

We acknowledge The UK Office for National Statistics (ONS) and NHS Digital for permission to use their data in this report. We also thank all staff within individual NHS trusts who collected and entered the data used in this study.

\section{References}

1. Hussey PS, de Vries H, Romley J, et al. A systematic review of health care efficiency measures. Health Serv Res 2009;44:784-805.

2. Papanicolas I, Mossialos E, Gundersen A, et al. Performance of UK National Health Service compared with other high income countries: observational study. BMJ 2019;367:16326.

3. Sutherland K and Levesque JF. Unwarranted clinical variation in health care: Definitions and proposal of an analytic framework. J Eval Clin Pract 2019 2019/05/29. DOI: 10.1111/jep.13181.

4. Harrison S. Urology: GIRFT Programme National Specialty Report. 2018. London, UK: GIRFT/NHS Improvement.

5. NHS Digital. Hospital Episode Statistics (HES) Analysis Guide. 2018. London, UK: NHS Digital.

6. Kontopantelis E, Doran T, Springate DA, et al. Regression based quasi-experimental approach when randomisation is not an option: interrupted time series analysis. BMJ 2015;350:h2750.

7. Linden A. Conducting interrupted time-series analysis for single- and multiple-group comparisons. Stata J 2015;15:480-500.

8. Cumby RE and Huizinga J. Testing the autocorrelation structure of disturbances in ordinary least squares and instrumental variables regressions. Econometrica 1992;60:185-195.

9. Baum CF and Schaffer ME. actest: Stata module to perform Cumby-Huizinga general test for autocorrelation in time series. Statistical Software Components

S457668. Department of Economics, Boston College, USA 2013.

10. Gray WK, Day J, Briggs TWR, et al. Transurethral resection of bladder tumour as day-case surgery: Evidence of effectiveness from the UK Getting it Right First Time (GIRFT) programme. J Clin Urol 2019; https://doi.org/10.1177/2051415819874562

11. Haleblian G, Kijvikai K, de la Rosette J, et al. Ureteral stenting and urinary stone management: a systematic review. J Urol 2008;179:424-430.

12. Turk C, Petrik A, Sarica K, et al. EAU Guidelines on Interventional Treatment for Urolithiasis. Eur Urol 2016;69:475-482.

13. Institute for Fiscal Studies and The Health Foundation. Securing the future: funding health and social care to the 2030s. 2018. London, UK: NHS Confederation

14. Timmins N. Tackling variations in clinical care: assessing the getting it right first time programme. 2017. London, UK: The King's Fund. 
15. Alawadi ZM, Leal I, Phatak UR, et al. Facilitators and barriers of implementing enhanced recovery in colorectal surgery at a safety net hospital: A provider and patient perspective. Surgery 2016;159:700-712.

16. Forsner T, Hansson J, Brommels M, et al. Implementing clinical guidelines in psychiatry: a qualitative study of perceived facilitators and barriers. BMC Psychiatry 2010;10:8.

17. Chaillet N, Dube E, Dugas M, et al. Identifying barriers and facilitators towards implementing guidelines to reduce caesarean section rates in Quebec. Bull World Health Organ 2007;85:791-797.

18. Shadish W, Cook T and Campbell D. Experimental and quasi-experimental designs for generalized causal inference. Boston, MA, USA: Houghton Mifflin

2002.

19. Penfold RB and Zhang F. Use of interrupted time series analysis in evaluating health care quality improvements. Acad Pediatr 2013;13:S38-44.

Figure legends

Figure 1: Change in day-case rate for transurethral resection of bladder tumour across the time series

Figure 2: Change in use of ureteric stents for stones across the time series

Figure 3: Change in percentage receiving ureteroscopy or extracorporeal shock wave lithotripsy within 30 days across the time series

Figure 4: Change in mean waiting times for transurethral resection of the prostate across the time series

Table 1. Interrupted time series analysis models

\begin{tabular}{lll}
\hline Intervention event & Parameter estimate & Significance \\
\hline Day-case surgery for TURBT & & \\
Starting value & $10.158(8.894$ to 11.422$)$ & - \\
Trend prior to visit & $0.105(0.067$ to 0.143$)$ & $<\mathbf{0 . 0 0 1}$ \\
Immediate change in level from pre to post visit & $-1.578(-3.493$ to 0.337$)$ & 0.105 \\
Post- visit trend & $0.281(0.171$ to 0.390$)$ & $<\mathbf{0 . 0 0 1}$ \\
Change in trend from pre to post visit & $0.176(0.060$ to 0.292$)$ & $\mathbf{0 . 0 0 3}$ \\
Use of ureteric stent & & \\
Starting value & $12.251(11.270$ to 13.233$)$ & - \\
Trend prior to visit & $0.105(0.068$ to 0.142$)$ & $<\mathbf{0 . 0 0 1}$ \\
Immediate change in level from pre to post visit & $-0.290(-1.703$ to 1.123$)$ & 0.984 \\
Post- visit trend & $-0.044(-0.110$ to 0.022$)$ & 0.193 \\
Change in trend from pre to post visit & $-0.148(-0.224$ to -0.073$)$ & $<\mathbf{0 . 0 0 1}$ \\
Use of ESWL or ureteroscopy & & \\
Starting value & $10.820(9.556$ to 12.085$)$ & - \\
Trend prior to visit & $-0.003(-0.048$ to 0.041$)$ & 0.877 \\
Immediate change in level from pre to post visit & $-0.077(-1.444$ to 1.290$)$ & 0.911 \\
Post- visit trend & $0.073(0.004$ to 0.024$)$ & $\mathbf{0 . 0 0 4}$ \\
Change in trend from pre to post visit & $0.077(0.010$ to 0.143$)$ & $\mathbf{0 . 0 2 4}$ \\
Waiting times for TURP & & \\
Starting value & $126.265(123.105$ to 129.425$)$ & $\mathbf{-}$ \\
Trend prior to visit & $0.310(0.181$ to 0.439$)$ & $<\mathbf{0 . 0 0 1}$ \\
Immediate change in level from pre to post visit & $-2.655(-8.577$ to 3.266$)$ & 0.373 \\
Post- visit trend & $0.261(-0.231$ to 0.752$)$ & 0.293 \\
&
\end{tabular}




\begin{tabular}{lll}
\hline Intervention event & Parameter estimate & Significance \\
\hline Change in trend from pre to post visit & $-0.050(-0.558$ to 0.458$)$ & 0.846 \\
\hline
\end{tabular}

\section{Hosted file}

Figure 1.docx available at https://authorea.com/users/317521/articles/447588-to-what-extenthave-key-recommendations-from-the-getting-it-right-first-time-programme-for-urology-inengland-been-implemented

\section{Hosted file}

Figure 2.docx available at https://authorea.com/users/317521/articles/447588-to-what-extenthave-key-recommendations-from-the-getting-it-right-first-time-programme-for-urology-inengland-been-implemented

\section{Hosted file}

Figure 3.docx available at https://authorea.com/users/317521/articles/447588-to-what-extenthave-key-recommendations-from-the-getting-it-right-first-time-programme-for-urology-inengland-been-implemented

\section{Hosted file}

Figure 4.docx available at https://authorea.com/users/317521/articles/447588-to-what-extenthave-key-recommendations-from-the-getting-it-right-first-time-programme-for-urology-inengland-been-implemented 